\title{
1. Phagocytosis of bacteria by olfactory ensheathing cells and Schwann cells
}

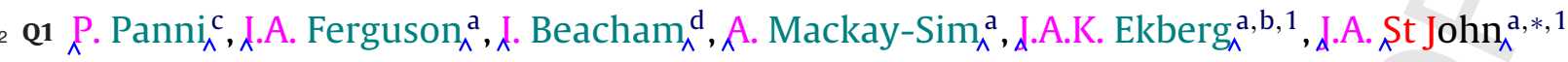 \\ a Eskitis Institute for Cell and Molecular Therapies, Griffith University, Nathan 4111, Queensland, Australia \\ b School of Biomedical Sciences, Queensland University of Technology, Brisbane 4000, Queensland, Australia \\ ${ }^{c}$ Neurosurgery Unit, San Raffaele Scientific Institute, Milano 20132, Italy \\ d Institute for Glycomics, Griffith University, Southport 4215, Queensland, Australia
}

\section{H I G H L I G H T S}

- Mouse olfactory ensheathing cells and Schwann cells phagocytose E. coli.

- Human OECs phagocytose E. coli.

- Peripheral glia show little capacity to phagocytose Burkholderia thailandensis.

- The results have implications for using glial cells in neural repair therapies.

\section{A R T I C L E I N F O}

\section{Article history:}

Received 19 November 2012

Received in revised form 21 January 2013

Accepted 31 January 2013

\section{Key words:}

Infection

Peripheral nervous system

Nasal cavity

E. coli

Burkholderia thailandensis

\begin{abstract}
A B S T R A C T
Opportunistic bacterial infections of the nasal cavity could potentially lead to infection of the brain if the olfactory or trigeminal nerves are colonised. The olfactory nerve may be a more susceptible route because primary olfactory neurons are in direct contact with the external environment. Peripheral glia are known to be able to phagocytose some species of bacteria and may therefore provide a defence mechanism against bacterial infection. As the nasal cavity is frequently exposed to bacterial infections, we hypothesised that the olfactory and trigeminal nerves within the nasal cavity could be subjected to bacterial colonisation and that the olfactory ensheathing cells and Schwann cells may be involved in responding to the bacterial invasion. We have examined the ability of mouse OECs and Schwann cells from the trigeminal nerve and dorsal root ganglia to phagocytose Escherichia coli and Burkholderia thailandensis in vitro. We found that all three sources of glia were equally able to phagocytose $E$. coli with $\lambda^{75-85 \%}$ of glia having phagocytosed bacteria within $24 \mathrm{~h}$, We also show that human OECs phagocytosed E. coli. In contrast, the mouse OECs and Schwann cells had little capacity to phagocytose B. thailandensis. Thus subtypes of peripheral glia have similar capacities for phagocytosis of bacteria but show selective capacity for the two different species of bacteria that were examined. These results have implications for the understanding of the mechanisms of bacterial infections as well as for the use of glia for neural repair therapies.
\end{abstract}

(c) 2013 Published by Elsevier Ireland Ltd.

\section{Introduction}

Various pathogens that infect the brain can enter the central nervous system via several potential neural routes including the peripheral nerves of the nasal cavity. The olfactory nerve perhaps offers the most accessible route as the primary olfactory neurons are in direct contact with the environment. The dendrites of primary olfactory neurons extend into the mucous layer that overlies the olfactory epithelium and the axons project into the olfactory bulb that lies within the central nervous system $[12,20]$. As the

\footnotetext{
* Corresponding author. Tel.: +61 73735 3660; fax: +61 737354255 .

E-mail address: j.stjohn@griffith.edu.au (J.A. St John).

1 Co-senior authors.
}

olfactory epithelium is often subjected to numerous infections which disrupt its integrity, opportunistic bacterial infections of the olfactory nerves may occur. Thus bacteria, including Burkholderia pseudomallei, are potentially able to enter and migrate within the olfactory nerve fascicles where they could then penetrate directly into the central nervous system and thereby bypass the blood-brain barrier [14]. However, the olfactory nerve is not the only potential route within the nasal cavity. The trigeminal nerve innervates the nasal cavity and projects into the central nervous system in close proximity to the olfactory nerves [5,16,17].

The glia of the olfactory system are the olfactory ensheathing cells (OECs) which surround the bundles of axons to form the olfactory nerves [2]. OECs have previously been shown to endocytose Escherichia coli in vitro $[9,19]$ as well as Streptococcus pneumonia [10]. It has been proposed that the phagocytic activity of the OECs 
is an important mechanism to combat bacterial infection although it has not yet been tested in vivo [9]. The glia of other peripheral nerves are Schwann cells. While both mouse and human Schwann cells have been shown to be infected by mycobacteria $[13,15,18]$, it has been reported that unlike OECs, exposure of Schwann cells to inactivated $E$. coli does not activate nuclear factor KB and Gro production suggesting that the mechanism by which OECs and Schwann cells react to E. coli is different. [19].

While the phagocytic ability of peripheral nerve glia within the nasal cavity may offer protection against bacterial infection, it has not been determined whether different subtypes of glia have similar abilities to phagocytose bacteria. In addition, as OECs and Schwann cells are being considered for neural repair therapies [11] and the potential capacity for phagocytosis of bacteria within an injury repair site may be of benefit, it is important to determine the phagocytic capacities of the different glia.

In the current study, we have examined the ability of OECs and Schwann cells from the trigeminal nerve and dorsal root ganglia to phagocytose E. coli and Burkholderia thailandensis in vitro. We found that all three sources of glia were equally able to phagocytose E. coli, but had little capacity to phagocytose B. thailandensis. These results suggest that peripheral glia have similar capacities for phagocytosis of bacteria but show selective capacity for different species of bacteria.

\section{Materials and methods}

\subsection{Animals}

For cultures of glia we used S100ß-DsRed transgenic mice [22] as the S100ß promoter drives expression of DsRed resulting in glia being visualised by DsRed expression. All procedures were carried out with the approval of the Griffith University Animal Ethics Committee under the guidelines of the Australian Commonwealth Office of the Gene Technology Regulator.

\subsection{Cell culture}

Postnatal day 7 pups were decapitated. The olfactory mucosa overlying the nasal septum was dissected out for preparations of olfactory ensheathing cells (OECs). Dorsal root ganglia (DRG) from the spinal cord was dissected out for DRG Schwann cells. The trigeminal nerve lying on the basal surface of the cranial cavity was dissected out for trigeminal Schwann cells. Explants of the different glia preparations were separately plated in wells that had been coated with Matrigel basement membrane matrix (BD Bioscience, $1: 10$ ) and were maintained in glia medium: Dulbecco's Modified Eagle Medium containing 10\% foetal bovine serum, G5 supplement (Gibco), gentamicin (Gibco, $50 \mathrm{mg} / \mathrm{mL}$ ) and L-glutamine $(200 \mu \mathrm{M})$ at $37{ }^{\circ} \mathrm{C}$ with $5 \% \mathrm{CO}_{2}$ for 5 days. Cells were replated into plastic 24 -well plates and grown to $80 \%$ confluency.

\subsection{Human OECS}

Frozen stocks of human OECs were previously prepared and characterised [4,7]. Frozen cells were thawed and grown in 24-well plastic plates in glia medium at $37^{\circ} \mathrm{C}$ with $5 \% \mathrm{CO}_{2}$ for 5 days and grown to $80 \%$ confluency.

\subsection{Bacteria preparation}

$E_{\wedge}$ coli (K-12 strain) and $B_{\wedge}$ thailandensis (E264) were grown in liquid Luria broth (LB) media with shaking or on LB agar plates. E. coli was autoclaved and labelled with fluorescein 5-isothiocyanate (FITC, Sigma) using a modification of the method of Hazenbos et al.
[8]. The bacteria were washed 5 times by centrifugation and resuspended in $5 \mathrm{~mL}$ of $0.1 \mathrm{M}$ sodium carbonate buffer, $\mathrm{pH} 8.6$ with final resuspension in $1 \mathrm{~mL}$ of buffer. FITC ( $1 \mathrm{mg} / \mathrm{mL}$ DMSO) was added at a ratio of $5 \mu \mathrm{L}$ to $1 \mathrm{~mL}$ of the bacteria in sodium carbonate buffer for $18 \mathrm{~h}$ at $4^{\circ} \mathrm{C}$; then washed by centrifugation/resuspension in $0.1 \mathrm{M}$ sodium carbonate buffer, $\mathrm{pH}$ 8.6. Labelling of $B_{\wedge}$ thailandensis with carboxyfluorescein diacetate (CFDA) was as described previously [3]; $1 \mathrm{~mL}$ of bacterial culture was pelleted by centrifugation, washed once with phosphate buffered saline (PBS) and resuspended in $500 \mu \mathrm{L}$ of $5 \mu \mathrm{M}$ carboxyfluorescein diacetate, succinimidyl ester (CFDA-SE, Molecular probes). The resuspended cells were incubated at $37^{\circ} \mathrm{C}$ for $30 \mathrm{~min}$, washed twice with PBS and resuspended in Hanks balanced salt solution (HBSS) prior to use. Bacteria concentration was determined from optical density at $600 \mathrm{~nm}$. E. coli and $B$. thailandensis were used at equal concentrations by optical density. Bacteria solutions were stored as frozen aliquots and freshly thawed for each experiment.

\subsection{Phagocytosis assays}

E. coli and B. thailandensis were added to the culture medium with the same bacterial load being added to all wells within each experiment as determined by spectrophotometry; experiments for quantification shown in Fig. 4 used half the bacterial load to enable easier quantification of the cells. After 6,18 or $24 \mathrm{~h}$ the medium was removed and the wells washed three times with HBSS to remove the majority of the extracellular bacteria. The cells were resuspended, centrifuged with repeated washes to eliminate residual extracellular bacteria. The cells were replated onto glass coverslips coated with Matrigel and maintained in the glia medium for 6 h. Cells were fixed for $4 \mathrm{~min}$ in $4 \%$ paraformaldehyde and cell nuclei were stained with $4^{\prime}, 6$-diamidino-2-phenylindole.

\subsection{Immunohistochemistry}

Human OECs were fixed with $4 \%$ paraformaldehyde for $10 \mathrm{~min}$, rinsed with PBS and then blocked with $2 \%$ bovine serum albumin (BSA) in PBS for $30 \mathrm{~min}$. The cells were incubated with mouse anti-human p75 neurotrophin receptor antibodies (Biosensis, $2.5 \mu \mathrm{g} / \mathrm{mL}$ ) in $2 \%$ BSA with PBS with $0.3 \%$ Triton-X for $1 \mathrm{~h}$, washed with PBS with Triton-X, and then incubated with secondary antibodies goat anti-mouse Alexa Fluor 488 (1:200; Invitrogen). Cell nuclei were stained with $\Lambda^{\prime}, 6$-diamidino-2-phenylindole.

\subsection{Imaging}

Lower power images were captured on an Olympus BX50 microscope or an Olympus IX70 inverted microscope fitted with fluorescence optics and a Spot camera and software. Higher magnification images were collected using an Olympus FV-1000 confocal microscope. Images were coloured balanced using Adobe Photoshop CS5 (Adobe Systems Incorporated) with the entire field of view being altered uniformly and figures were compiled using Adobe Illustrator CS5 (Adobe Systems Incorporated).

\subsection{Quantification and statistics}

The number of DsRed glia that contained bacteria and the total number of DsRed glia were counted in the fields of view obtained with a $10 \times$ objective. One field of view for each treatment with four replicates was counted, with at least 40 cells per field of view. Statistical analysis was performed using ANOVA. 


\section{Results}

To determine whether mouse olfactory ensheathing cells (OECs), Schwann cells from the trigeminal nerve and Schwann cells from dorsal root ganglia (DRG) have similar capacities to phagocytose bacteria we prepared cultures of the different glia and incubated them with $E$. coli. After $6 \mathrm{~h}$ of incubation, the cultures of all three types of mouse glia exhibited some cells that had phagocytosed bacteria (Fig. 1). To enable better visualisation of the internalised bacteria, the cultures were washed, trypsinised and replated to remove the extracellular bacteria (Fig. 1G). Some cells had small accumulations of bacteria within cytoplasm while others within the same field of view had large accumulations of $E$. coli (Fig. 1A, C, and E). After $24 \mathrm{~h}$ incubation with E. coli, the majority of glia contained fluorescent bacteria within the cytoplasm with many cells having large accumulations of bacteria (Fig. 1B, D, and F). We quantified the proportion of DsRed-positive glia that contained the fluorescent bacteria. After $24 \mathrm{~h}$ incubation with $E$. coli, $75-85 \%$ of glia within each culture contained bacteria (Fig. $1 \mathrm{H}$ ). Statistical analysis showed that OECs, trigeminal Schwann cells and DRG Schwann cells had similar capacities to phagocytose E. coli (Fig. 1G, no significant difference, $p>0.48$ ). Other cells within the culture were present that were weakly positive for DsRed or did not express DsRed and some of these cells also contained bacteria (Fig. 1A, arrowhead).

Time-lapse imaging of mouse OECs that were incubated with FITC-labelled E. coli demonstrated that $E$. coli that was external to the OECs became internalised over time (Fig. 2 and supplemental movie 1 ). The E. coli that had been phagocytosed appeared as yellow rods within the shaft of the OECs. It was also apparent that there was a reduction of free floating E. coli (bright green dots in Fig. 2) over the time-lapse imaging period which supports the notion that the $E$. coli was phagocytosed.

We next confirmed that the E. coli was phagocytosed and contained within the cytoplasm of the glia. High magnification confocal microscopy confirmed that the bacteria were within the cytoplasm of mouse OECs, trigeminal Schwann cells and DRG Schwann cells (Fig. 3A-C). A three-dimensional reconstruction and rotation of an

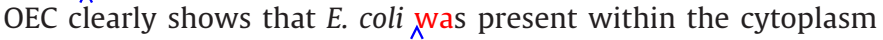
(supplemental Movie 2).

As OECs are being trialled for neural repair therapies, we also examined human OECs that had been previously obtained and characterised from nasal biopsies [4,7]. The human OECs, which were positive for the OEC marker p75 neurotrophin receptor (Fig. 3D inset), also phagocytosed E. coli within 24 h (Fig. 3D).

We next determined whether the peripheral glia were able to phagocytose bacteria other than E. coli. Like E. coli, B. thailandensis is a Gram-negative bacillus. Due to its similarity to the pathogen B. pseudomallei, it is used as a model for B. pseudomallei infection $[1,21]$. Mouse OECs, trigeminal Schwann cells, and DRG Schwann cells were incubated with $E$. coli or B. thailandensis at half the bacterial load of the earlier experiments in order to enable easier quantification of the cells and to minimise the number of cells that contained numerous bacteria. While E. coli was phagocytosed within each of the peripheral glia cultures, $B$. thailandensis was rarely observed within the cytoplasm of the glia and when it was, only a single rod was present (Fig. 4). The number of cells that contained either E. coli or $B$. thailandensis was counted and significantly fewer cells ( $p<0.001$ ANOVA and $p<0.05-0.001$ post hoc tests) had internalised $B$. thailandensis in comparison to $E$. coli.

\section{Discussion}

Here we have shown that peripheral glia from the olfactory nerve, the trigeminal nerve and dorsal root ganglia have similar
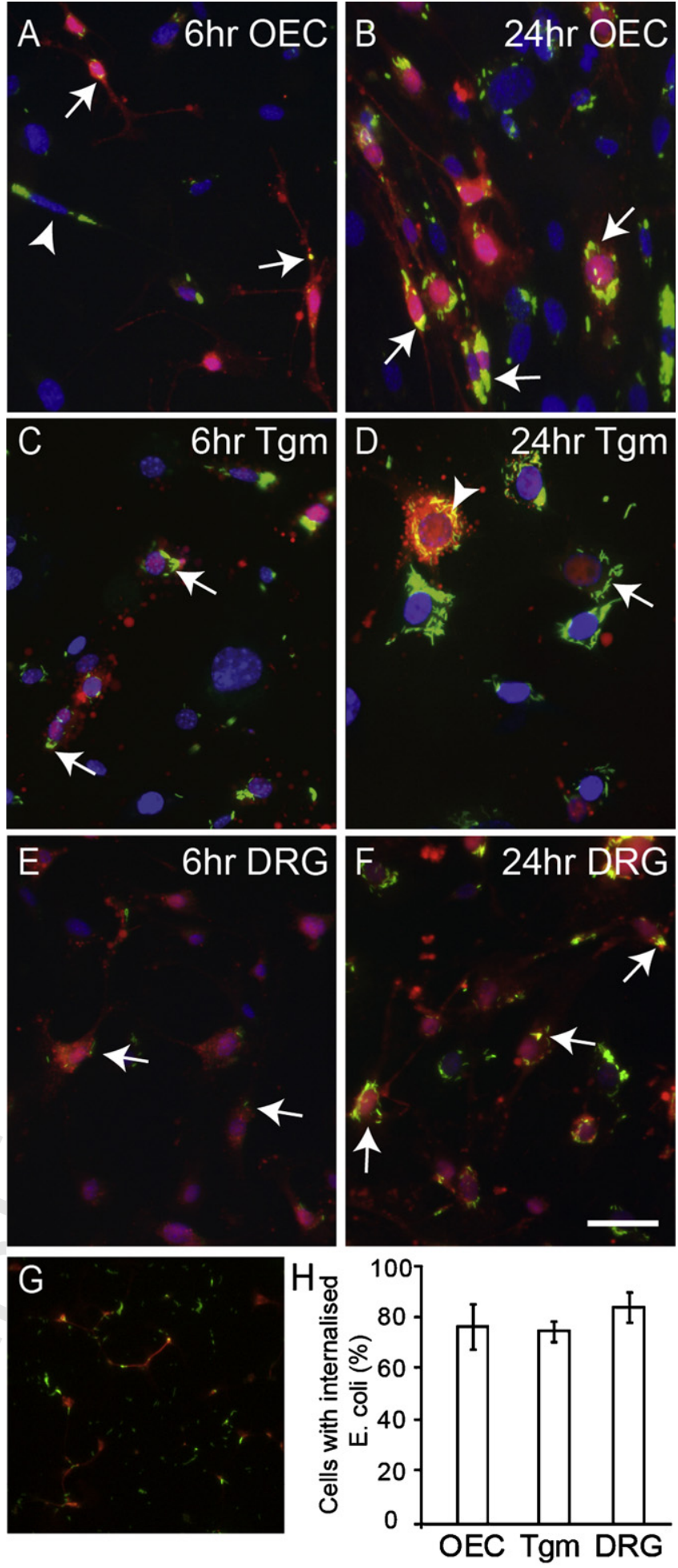

Fig. 1. Mouse OECs and Schwann cells phagocytose E. coli. OECs (A and B) Schwann cells from the trigeminal nerve ( $C$ and $D$ ) and Schwann cells from the dorsal root ganglia (E and F) were incubated with E. coli labelled with FITC (green, arrows) for $6 \mathrm{~h}$ ( $\mathrm{A}$, $\mathrm{C}$, and $\mathrm{E}$ ) and $24 \mathrm{~h}$ (B, D, and F). All glia express fluorescent DsRed protein, nuclei are stained with DAPI (blue). Some cells that were not DsRed-positive contained E. coli (arrowhead in A). A large flattened OEC (arrowhead) that had internalised E. coli is shown in $(D)$. (G) Example of the bacterial load during the assay; cells were then washed with trypsin to remove extracellular bacteria, resuspended and replated for analysis. (H) Quantification of the proportion of DsRed glia that contained green fluorescent $E$. coli after $24 \mathrm{~h}$ incubation. ANOVA analysis $p>0.48, n=173-544$ cells over 4 replicates. Scale bar $=50 \mu \mathrm{m}$ in $(A), 40 \mu \mathrm{m}$ in $(B-F)$, and $200 \mu \mathrm{m}$ in (G). (For Q2 interpretation of the references to colour in this figure legend, the reader is referred to the web version of this article.) 

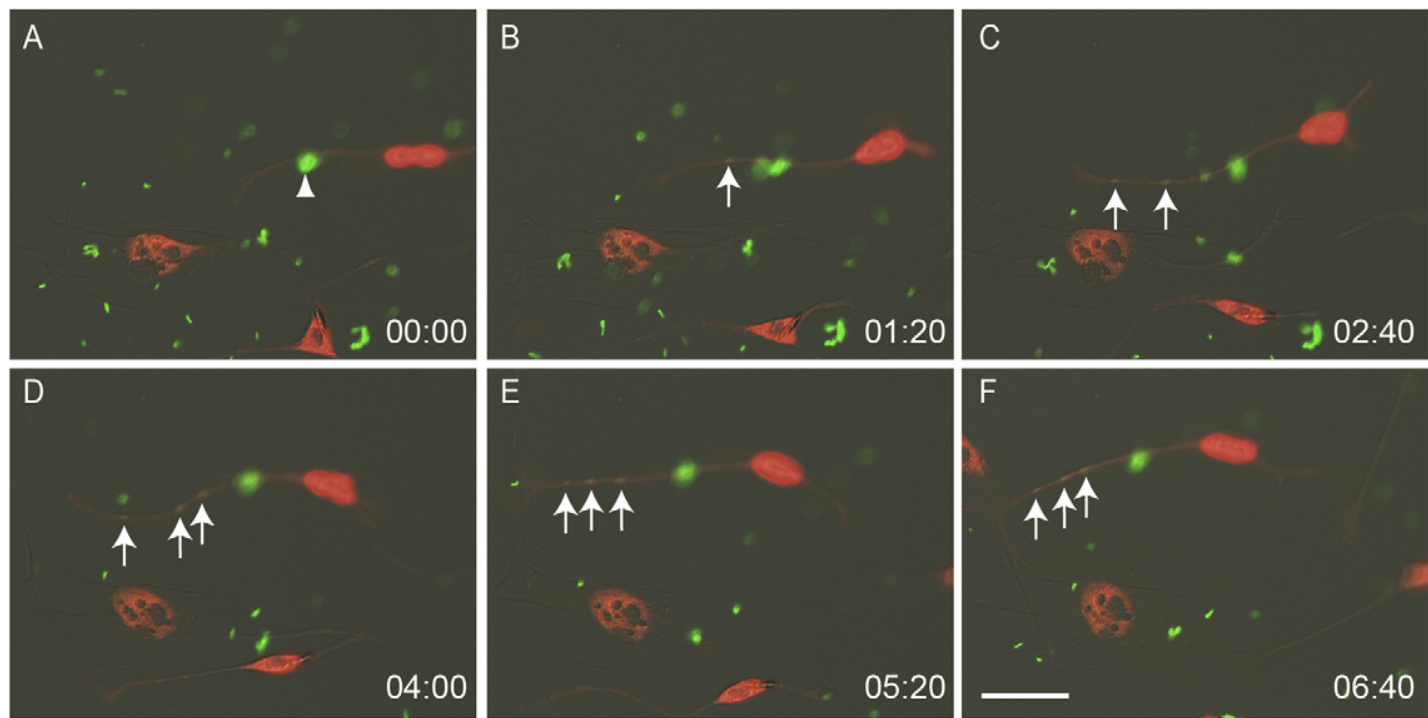

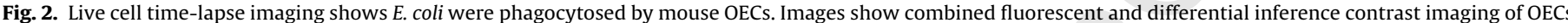

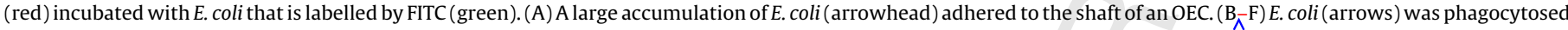

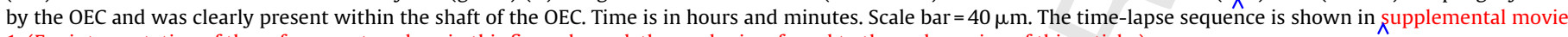
1. (For interpretation of the references to colour in this figure legend, the reader is referred to the web version of this article.)
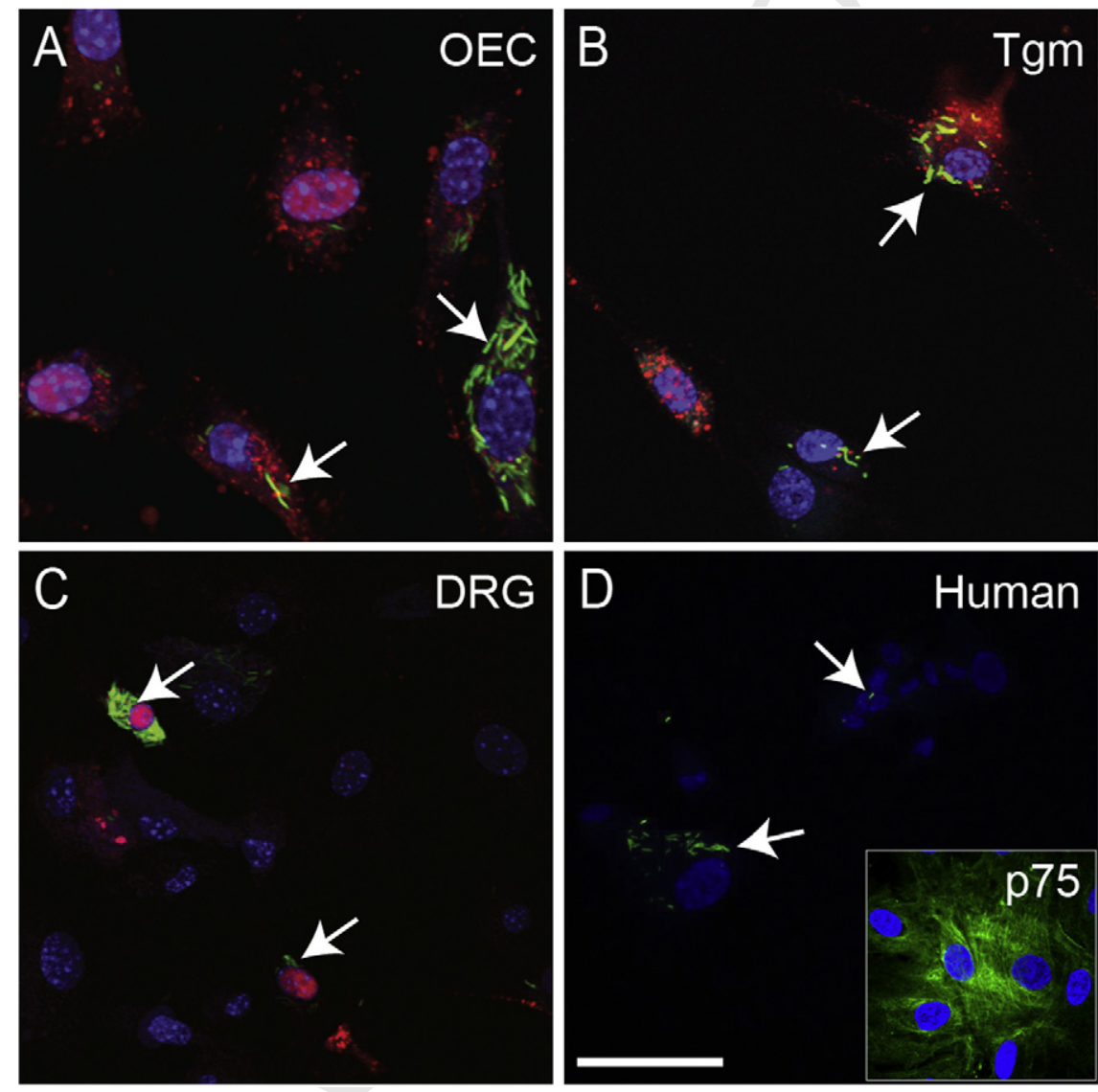

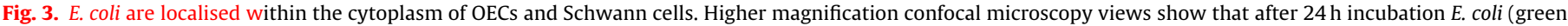

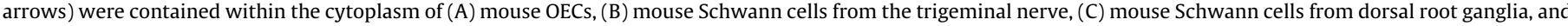

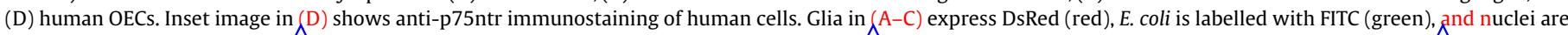

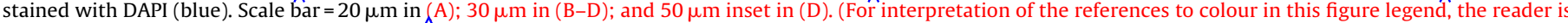
referred to the web version of this article.) 

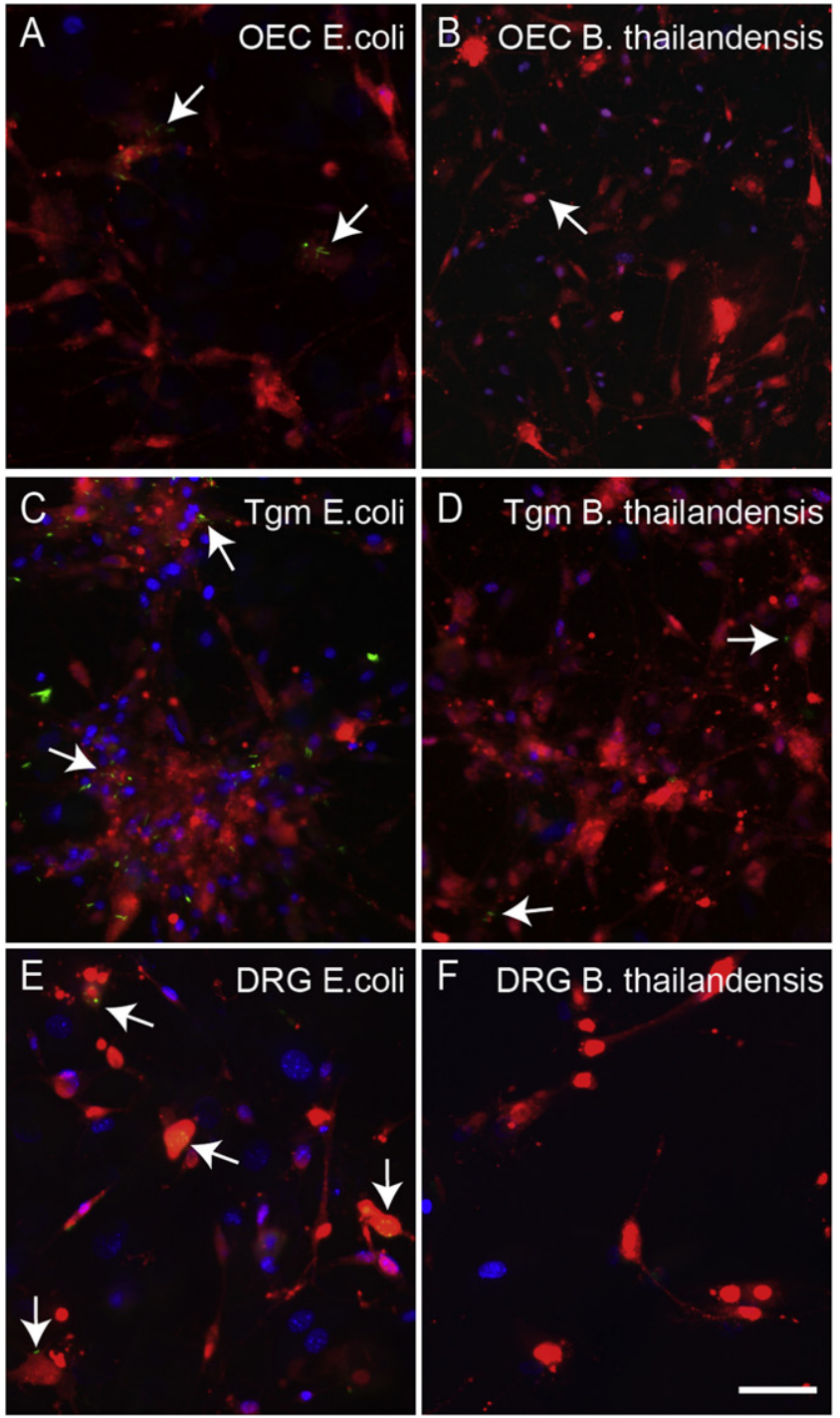

$$
\text { G }
$$

\section{政}

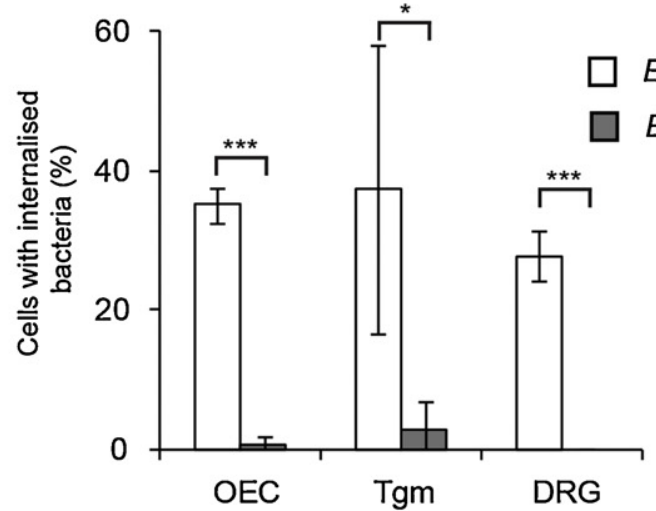

Fig. 4. Mouse OECs and Schwann cells phagocytose E. coli more avidly than B. thailandensis. Mouse OECs ( $A$ and $B$ ), Schwann cells from the trigeminal nerve ( $C$ and $D)$ and Schwann cells from dorsal root ganglia ( $E$ and $F$ ) were incubated for $18 \mathrm{~h}$ with E. coli (A, C, and E) or B. thailandensis (B, D, and F). Arrows point to bacteria internalised within the glia. Bacteria are labelled with green fluorescence, nuclei are stained with DAPI (blue). Scale bar $=80 \mu \mathrm{m}$ in (A-D) and $50 \mu \mathrm{m}$ in (E-F). Quantification of the percentage of cells with internalised $E$. coli (unfilled columns) or $B$. thailandensis (filled columns); $n=20-60$ cells in each of three fields of view; $p<0.0001$ ANOVA, and ${ }^{*} p<0.05$, ${ }^{* * *} p<0.001$ for post hoc $t$-tests. (For interpretation of the references to colour in this figure legend, the reader is referred to the web version of this article.) capacities to phagocytose $E$. coli. In stark contrast, when challenged with $B$. thailandensis all three types of glia were only able to phagocytose very small quantities of the bacteria. These results show that the three peripheral glia examined have a similar, but selective, ability to phagocytose bacteria.

It has been previously shown that OECs can phagocytose E. coli and that Toll-like receptor 4 mediates the binding of the bacteria to OECs $[9,19]$. Interestingly, it was reported that rat Schwann cells purified from the brachial plexus and sciatic nerve did not respond to $E$. coli after $2 \mathrm{~h}$ incubation [19]; although perhaps with longer incubation the Schwann cells may have phagocytosed the E. coli. In our hands, mouse Schwann cells from the trigeminal nerve and dorsal root ganglia both avidly phagocytosed $E$. coli when incubated for at least $6 \mathrm{~h}$, The reason for this difference may reflect differences between rodent species or indicate that subtypes of Schwann cells have differing capacities.

Some cells within the cultures did not express DsRed; these cells are likely to be fibroblasts or immature glia which are known to express similar markers and make identification difficult [6]. However, the presence of these cells may contribute to the ability of the glia cells to phagocytose the bacteria by secreting inflammatory factors. The interaction with other cells could be explored in future experiments.

Infections of Burkholderia pseudomallei have previously been localised to the nasal cavity and mucosal tissue with distinct infections within the olfactory nerve and olfactory bulb. Despite extensive infection of the nasal cavity, colonisation of the trigeminal nerve or the brain stem was not observed, though not excluded, in that study which suggested that infection of the brain was likely to occur via the olfactory nerve [14]. In the current study, we used B. thailandensis as it is often used as a non-pathogenic model for B. pseudomallei [21]. We found that the different glia largely did not respond to $B$. thailandensis and little phagocytosis occurred. Thus we were unable to determine whether OECs and trigeminal Schwann cells have differing capacities to phagocytose Burkholderia species. Nevertheless, it is clear that peripheral glia can distinguish between species of bacteria which may be due to differential carbohydrate expression by bacteria and complementary receptor expression by the glia [10].

As OECs and Schwann cells are being trialled for neural repair therapies [11] our results are of interest since they show that the different glia have similar capacities for phagocytosis. We have also shown that similar to mouse OECs, human OECs phagocytosed E. coli. With any surgical intervention there is a risk of contamination. Therefore transplanting cells that are known to actively phagocytose bacteria only adds to the benefits of using peripheral glia for neural repair therapies.

\section{Conclusion}

We have shown that OECs and Schwann cells from the trigeminal nerve and dorsal root ganglia have similar capacities to phagocytose $E$. coli, but are essentially unable to phagocytose $B$. thailandensis.

\section{Acknowledgements}

This work was supported by funding from Griffith University to J.St.J and J.E.; from the Australian Government Department of Health and Ageing and the Queensland Government Smart State National and International Research Alliances Programme to A.M.S., by an Australian Research Council Postdoctoral Fellowship to J.E, and by national project funding from Regione Lombardia to P.P.
225 


\section{Appendix A. Supplementary data}

Supplementary data associated with this article can be found, in the online version, at http://dx.doi.org/10.1016/ j.neulet.2013.01.052.

\section{References}

[1] P.J. Brett, D. DeShazer, D.E. Woods, Burkholderia thailandensis sp. nov., a Burkholderia pseudomallei-like species, Int ${ }_{\Lambda}$ J. Syst. Bacteriol. 1 (48 Pt.) (1998) 317-320.

[2] M.I. Chuah, C. Au, Olfactory Schwann cells are derived from precursor cells in the olfactory epithelium, J Neurosci. Res. 29 (1991) 172-180.

[3] C.J. Day, J. Tiralongo, R.D. Hartnell, C.A. Logue, J.C. Wilson, M. von Itzstein, V. Korolik, Differential carbohydrate recognition by Campylobacter jejuni strain 11168: influences of temperature and growth conditions, PLoS ONE 4 (2009) e4927.

[4] F. Feron, C. Perry, J.J. McGrath, A. Mackay-Sim, New techniques for biopsy and culture of human olfactory epithelial neurons, Arch $\wedge$ Otolaryngol. Head Neck Surg. 124 (1998) 861-866.

[5] T.E. Finger, B. Bottger, Peripheral peptidergic fibers of the trigeminal nerve in the olfactory bulb of the rat, J Comp. Neurol. 334 (1993) 117-124.

[6] V. Garcia-Escudero, A. Garcia-Gomez, E. Langa, M.J. Martin-Bermejo, R. Ramirez-Camacho, J.R. Garcia-Berrocal, M.T. Moreno-Flores, J. Avila, F. Lim, Patient-derived olfactory mucosa cells but not lung or skin fibroblasts mediate axonal regeneration of retinal ganglion neurons, Neurosci $\wedge_{\Lambda}$ Lett. 509 (2012) 27-32.

[7] C.A. Gorrie, I. Hayward, N. Cameron, G. Kailainathan, N. Nandapalan, R. Sutharsan, J. Wang, A. Mackay-Sim, P.M. Waite, Effects of human OEC-derived cell transplants in rodent spinal cord contusion injury, Brain Res 1337 (2010) 8-20.

[8] W.L. Hazenbos, B.M. van den Berg, J.W. van't Wout, F.R. Mooi, R. van Furth, Virulence factors determine attachment and ingestion of nonopsonized and opsonized Bordetella pertussis by human monocytes, Infect Immun. 62 (1994) $4818-4824$.

[9] J.Y. Leung, J.A. Chapman, J.A. Harris, D. Hale, R.S. Chung, A.K. West, M.I. Chuah, Olfactory ensheathing cells are attracted to, and can endocytose, bacteria, Cell Mol Life Sci. 65 (2008) 2732-2739.

[10] H. Macedo-Ramos, F.S. Campos, L.A. Carvalho, I.B. Ramos, L.M. Teixeira, W. De Souza, L.A. Cavalcante, W. Baetas-da-Cruz, Olfactory ensheathing cells as putative host cells for Streptococcus pneumoniae: evidence of bacterial invasion via mannose receptor-mediated endocytosis, Neurosci $\wedge$ Res. 69 (2011) 308-313.

[11] A. Mackay-Sim, J.A. St. John, Olfactory ensheathing cells from the nose: clinica application in human spinal cord injuries, Exp Neurol. 229 (2011) 174-180.

[12] M. Marin-Padilla, M.R. Amieva, Early neurogenesis of the mouse olfactory nerve: golgi and electron microscopic studies, $\mathrm{J}_{\Lambda}$ Comp. Neurol. 288 (1989) 339-352.

[13] K.A. Mattos, V.G. Oliveira, H. D’Avila, L.S. Rodrigues, R.O. Pinheiro, E.N. Sarno, M.C. Pessolani, P.T. Bozza, TLR6-driven lipid droplets in Mycobacterium lepraeinfected Schwann cells: immunoinflammatory platforms associated with bacterial persistence, J Immunol. 187 (2011) 2548-2558.

[14] S.J. Owen, M. Batzloff, F. Chehrehasa, A. Meedeniya, Y. Casart, C.A. Logue, R.G. Hirst, I.R. Peak, A. Mackay-Sim, I.R. Beacham, Nasal-associated lymphoid tissue and olfactory epithelium as portals of entry for Burkholderia pseudomallei in murine melioidosis, J Infect. Dis. 199 (2009) 1761-1770.

[15] H. Saito, H. Tomioka, K. Sato, T. Watanabe, Abilities of human oligodendroglial cells and mouse Schwann cells to phagocytose Mycobacterium leprae and other mycobacteria, Infect Immun. 51 (1986) 157-162.

[16] M.L. Schaefer, B. Bottger, W.L. Silver, T.E. Finger, Trigeminal collaterals in the nasal epithelium and olfactory bulb: a potential route for direct modulation of olfactory information by trigeminal stimuli, J Comp. Neurol. 444 (2002) 221-226.

[17] W.L. Silver, T.E. Finger, The anatomical and electrophysiological basis of peripheral nasal trigeminal chemoreception, Ann $\wedge$ N. Y. Acad. Sci. 1170 (2009) 202-205.

[18] R.M. Teles, S.R. Krutzik, M.T. Ochoa, R.B. Oliveira, E.N. Sarno, R.L. Modlin, Interleukin-4 regulates the expression of CD209 and subsequent uptake of Mycobacterium leprae by Schwann cells in human leprosy, Infect $\wedge^{\text {Immun. }} 78$ (2010) 4634-4643.

[19] A.J. Vincent, D.L. Choi-Lundberg, J.A. Harris, A.K. West, M.I. Chuah, Bacteria and PAMPs activate nuclear factor kappaB and Gro production in a subset of olfactory ensheathing cells and astrocytes but not in Schwann cells, Glia 55 (2007) 905-916.

[20] J.G. Whitesides III, A.S. LaMantia, Differential adhesion and the initial assembly of the mammalian olfactory nerve, J Comp. Neurol. 373 (1996) 240-254.

[21] W.J. Wiersinga, A.F. de Vos, R. de Beer, C.W. Wieland, J.J. Roelofs, D.E. Woods, T. van der Poll, Inflammation patterns induced by different Burkholderia species in mice, Cell Microbiol 10 (2008) 81-87.

[22] L.C. Windus, C. Claxton, C.L. Allen, B. Key, J.A. St John, Motile membrane protrusions regulate cell-cell adhesion and migration of olfactory ensheathing glia, Glia 55 (2007) 1708-1719. 